\title{
The Role of Syndrome Differentiation in the Clinical Efficacy of Punica Granatum on Patients with Ulcerative Colitis
}

Mohammadali Kamali', Mahmoud Khodadoost ${ }^{1}$, Hamid Tavakoli², Mohammad Kamalinejad ${ }^{3}$, Latif Gachkar ${ }^{4}$, Payman Adibi Mojtaba Heydari ${ }^{5}$

1Department of Traditional Medicine, School of Traditional Medicine, Shahid Beheshti University of Medical Sciences, Tehran, Iran;

2Integrative Functional Gastroenterology Research Center, Isfahan University of Medical Sciences, Isfahan, Iran; ${ }^{3}$ School of Pharmacy, Shahid Beheshti University of Medical Sciences, Tehran, Iran;

${ }^{4}$ Infectious Disease and Tropical Medicine Research Center, Shahid Beheshti University of Medical Sciences, Tehran, Iran;

${ }^{5}$ Research Center for Traditional Medicine and History of Medicine, Shiraz University of Medical Sciences, Shiraz, Iran

\begin{abstract}
Background: The present study investigated the difference between therapeutic responses of hot and cold temperament patients (based on traditional Persian medicine) with ulcerative colitis to pomegranate peel extract.

Methods: Seventy-eight patients with moderate ulcerative colitis based on Lichtiger Colitis Activity Index (LCAI) criteria were randomized to receive an aqueous extract of the Punica granatum peel (6 gram per day) or placebo for four weeks. They were assessed before and after the intervention in terms of symptoms by LCAI scoring system. The results were compared in two therapeutic groups based on the patient s' temperament (cold and hot) which were diagnosed based on a previously validated questionnaire.
\end{abstract}

Results: Therapeutic response was significantly higher in patients with hot temperament compared to patients with cold temperament in the P. granatum group $(1.91 \pm 0.492$ vs. $-0.500 \pm 0.500, \mathrm{P}=0.029$ ).

Conclusion: This study showed the importance of considering syndrome differentiation and temperament in interpreting the effect of $\mathrm{P}$. granatum peel extract on ulcerative colitis.

Keywords $\bullet$ Medicine $\bullet$ Traditional $\bullet$ Punicaceae $\bullet$ Colitis $\bullet$ Ulcerative 